\title{
Nanoanalytical Electron Microscopy Reveals A Sequential Mineralization Process Involving Carbonate-Containing Amorphous Precursors
}

\author{
Kharissa Nitiputri ${ }^{1,2,3}$, Quentin M. Ramasse ${ }^{4}$, Hélène Autefage ${ }^{1,2,3}$, Catriona M. McGilvery ${ }^{1}$,

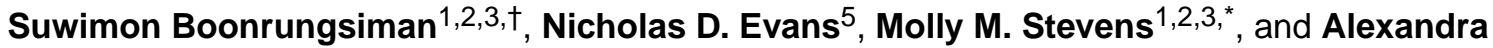 \\ E. Porter ${ }^{1, *}$ \\ ${ }^{1}$ Department of Materials, Imperial College London, London SW7 2AZ UK \\ ${ }^{2}$ Department of Bioengineering, Imperial College London, London SW7 2AZ UK \\ ${ }^{3}$ Institute of Biomedical Engineering, Imperial College London, London SW7 2AZ UK \\ ${ }^{4}$ SuperSTEM Laboratory, SciTech Daresbury Campus, Daresbury, WA4 4AD \\ ${ }^{5}$ Department of Bioengineering and Institute for Life Sciences, University of Southampton, \\ Southampton, SO17 1BJ
}

\section{Abstract}

\begin{abstract}
A direct observation and an in-depth characterization of the steps by which bone mineral nucleates and grows in the extracellular matrix during the earliest stages of maturation, using relevant biomineralization models as they grow into mature bone mineral, is an important research goal. To better understand the process of bone mineralization in the extracellular matrix, we used nanoanalytical electron microscopy techniques to examine an in vitro model of bone formation. This study demonstrates the presence of three dominant $\mathrm{CaP}$ structures in the mineralizing osteoblast cultures: $<80 \mathrm{~nm}$ dense granules with a low calcium to phosphate ratio $(\mathrm{Ca} / \mathrm{P})$ and crystalline domains; calcium phosphate needles emanating from a foci: "needle-like globules" (100-300 nm in diameter); and mature mineral, both with statistically higher $\mathrm{Ca} / \mathrm{P}$ compared to that of the dense granules. Many of the submicron granules and globules were interspersed around fibrillar structures containing nitrogen, which are most likely the signature of the organic phase. With high spatial resolution electron energy loss spectroscopy (EELS) mapping, spatially resolved maps were acquired showing the distribution of carbonate within each mineral structure. The carbonate
\end{abstract}

\footnotetext{
"Corresponding Author, To whom all correspondence should be addressed: Professor Molly M. Stevens, Imperial College London, Department of Materials, Royal School of Mines, London SW7 2AZ, UK. Tel: +44 (0) 20 7594 6804; m.stevens@imperial.ac.uk and Dr. Alexandra Porter, Imperial College London, Department of Materials, Royal School of Mines, London SW7 2AZ, UK. Tel: +44 (0) 207594 9691, a.porter@imperial.ac.uk.

$\uparrow$ Present Addresses

National Nanotechnology Center (NANOTEC), National Science and Technology Development Agency (NSTDA), Pathumthani, Thailand

Author Contributions

K.N., C.M.M. and Q.R. carried out the experiments. Q.R. conducted the SuperSTEM EELS experiments. C.M.M. and K.N. conducted the TEM work at Imperial College. K.N., Q.R., A.E.P. and C.M.M. analysed the data. S.B. and H.A. carried out the cell culture experiments. A.E.P supervised the project. A.E.P. and M.M.S. designed the project. Q.R, A.E.P, K.N., H.A., C.M.M. and N.E. wrote the paper. A.E.P, H.A. and N.E revised the manuscript.

Conflict of interest: The authors declare no competing financial interest.
} 
was located in the middle of the granules, which suggested the nucleation of the younger mineral starts with a carbonate-containing precursor and that this precursor may act as seed for growth into larger, submicron-sized, needle-like globules of hydroxyapatite with a different stoichiometry. Application of analytical electron microscopy has important implications in deciphering both how normal bone forms and in understanding pathological mineralization.

\section{Keywords}

Carbonate; Mineralization; Electron Energy Loss Spectroscopy; Bone; Calcium Phosphate Precursor

Bone is a nano-composite material made of poorly crystalline, carbonated apatite-like crystals intimately associated with the collagen fibrils. 1 The mechanism of bone mineralization is controversial, but it is usually believed to involve precipitation and crystal growth of transient calcium phosphate $(\mathrm{CaP})$ precursors within the collagen fibrils.2,3 There has been much debate in the literature about whether $\mathrm{CaP}$ precursor phases are delivered to the collagen and, if so, what the nature of these $\mathrm{CaP}$ precursor phases may be and whether these precursor phases wholly account for mineralization of the collagen fibrils.4,5 Amorphous mineral precursors, such as amorphous calcium phosphate (ACP), have been suggested to be a universal transient precursor to mature mineral in vertebrates and invertebrates.2-4, 6-8 Previous acellular studies showed that ACP could be converted to crystalline apatite in vitro in a solution containing calcium and phosphate complexes.9-12 In these studies, which incorporate isolated and reconstructed collagen fibrils, the $\mathrm{CaP}$ particles nucleated within the collagen fibrils and later grew into elongated crystals within the fibrils, a process directed by proteins. 13 In contrast, in the absence of extracellular matrix (ECM), $\mathrm{CaP}$ pre-nucleation species composed of calcium triphosphate ion-association complexes $(\mathrm{Ca} / \mathrm{P}=0.3-0.4)$ were found to first form a polymeric chain before condensing to sphericallyshaped ACP.

Acellular systems, however, still do not faithfully reflect how mineralization occurs in organisms and need to be supported by investigation of biological samples. A full understanding of this process is very important as uncontrolled precipitation of mineral in collagenous ECM, which is present in many mammalian tissues, including skin and blood vessels, may have severe pathological consequences.14,15 Therefore animals have evolved complex, cell-orchestrated mechanisms to control mineral deposition, ensuring it happens only in appropriate places such as the skeleton. Central to this process are osteoblasts, which have been proposed to promote and control deposition of mineral in the bones.4,16 To understand how osteoblasts control mineral deposition, cell culture models have been used to try and understand the process by which mineral is delivered from osteoblasts to the collagen.4,16 We16 and others4 have previously found strong evidence to suggest early mineral precursors originate intracellularly from mineral vesicles subsequently secreted by osteoblasts in proximity to a collagenous ECM. We found the existence of calcium-rich intracellular vesicles that were hypothesized to provide the appropriate environment for the initiation of the $\mathrm{CaP}$ mineralization. These amorphous $\mathrm{CaP}$ particles formed inside the cells are then released from the cells inside vesicles4,16 where they deposit in the ECM4 and 
undergo crystallization. Calcium-polyphosphate complexes, present in electron-dense intracellular vesicles, have been proposed as a source for intracellular phosphate, as they allow phosphate accumulation and can be hydrolytically degraded by the alkaline phosphatase, ensuring enzymatic control of mineral deposition.17-19

Supporting a role for a controlled crystallisation of $\mathrm{CaP}$ from cell derived vesicles, work in zebrafish, (using a combination of synchrotron micro-beam X-ray diffraction, small-angle $\mathrm{x}$ ray scattering and cryo-scanning electron microscopy (cryo-SEM) of frozen hydrated and freeze dried tissues) showed that the ECM contained globular entities, 200-500 nm in diameter, possibly composed of 10-15 nm spheres.2 Using larval zebrafish models, Akiva et al. have recently identified, in vivo, micrometer-sized mineral particles at distance from the bone and displaying Raman spectral signatures of a mixture of ACP and OCP-like phases.20 These results support Crane et al.'s previous work, which identified by Raman spectroscopy "modified OCP" (and possibly ACP) as transient CaP precursors for the formation of carbonated apatite in an ex vivo murine intramembranous mineralization model.21

The methods applied in these reports2,20,21 preserve mineral chemistry and morphology but do not have the spatial or chemical resolution to analyze the morphological and chemical relationship between the highly disordered $\mathrm{CaP}$ granules and the needle-like globules (or any intermediate structures). This information could provide an improved understanding of the physicochemical format in which the mineral is delivered to the collagen, and how the mineral evolves over time to form mature bone mineral.

High resolution spatially resolved analysis showing the steps by which the $\mathrm{CaP}$ precursor phases evolve both chemically and morphologically during the early stages of mineralization is missing from the literature. Very few techniques are available which provide spatiallyresolved information about the chemistry and coordination environment of the different physicochemical formats of $\mathrm{CaP}$ in mineralizing tissues.

To address this we have combined scanning transmission electron microscopy (STEM) with electron energy-loss spectroscopy (EELS), as well as high-resolution transmission electron microscopy (HRTEM), to analyze anhydrously preserved mineralizing osteoblast cultures. Utilization of an anhydrous method avoids aqueous solution-induced mineral phase transformations 3 and preserves the crystallinity of the bone apatite.22,23 This combination of techniques provides high spatial resolution (probe size of $\sim 1 \AA$, allowing the 'effective' resolution in datasets to be governed by the magnification and pixel size chosen rather than being limited by optical parameters) analytical information about the step-by-step process by which the mineral grows into mature poorly crystalline carbonated hydroxyapatite in the ECM. Specifically variations between the morphology, chemistry and coordination environment of the different formats of mineral detected within the nodules were analyzed to provide a link between how the $\mathrm{CaP}$ precursor granules nucleate, transform and grow into mature mineral crystals. To achieve this, we used a primary mouse calvarial osteoblast cell culture model. Calvarial osteoblasts form mineralized nodules after culture in the presence of osteogenic supplements with many of the structural features of mature bone, and represent a well-understood, controlled in vitro system for studying the mechanism by which bone cells control mineral deposition.16,24 


\section{Results and Discussion}

\section{ECM-associated mineral deposits vary in size and crystallinity and may represent different stages of maturity}

We first investigated the morphology and crystallinity of mineral deposits forming in bone nodules after culturing neonatal mouse calvarial osteoblasts for 28 days in osteogenic differentiation medium. As shown in Figure 1 and previously in our published work,(16) nodules were characterized by the presence of cells interspersed with large volumes of partially mineralized collagenous ECM (Figure 1A). Selected area electron diffraction (SAED) patterns taken from large mineralized areas (Figure 1B) are typical of polycrystalline hydroxyapatite (HA), consistent with the known hexagonal crystal form of $\mathrm{HA}(25)(\mathrm{a}=9.37 \AA$ and $\mathrm{c}=6.88 \AA$ ) (inset Figure 1B). In addition to these large mineralized deposits, smaller clusters of mineral were also observed in the ECM (Figure 1B).

For compositional analyses, we analyzed the mineral morphologies, which dominated the ECM, using high-angle annular dark field scanning transmission electron microscopy (HAADF-STEM). Here, the electron beam is condensed down to a probe and rastered across the sample to build up an image. Because of the small electron probe size $(\sim 1 \AA)$, high resolution images of the mineral structures can be obtained and can be combined with analytical techniques to give chemical information on the nanoscale. The mineral morphologies selected have been described in other publications on the progression of bone mineralization over time in situ.(2) For simplification, the three representative mineral structures found within the ECM were categorized as smaller "dense granules" with disordered morphologies ( $<80 \mathrm{~nm}$ in diameter; Figure 1C); calcium phosphate needles emanating from a focus: "needle-like globules" (100-300 nm in diameter; Figure 1D) and aligned needle-like mineral: "mature mineral" (Figure 1E). The smaller mineral clusters were composed of dense granules (Figure S2a,b) and needle-like globules emanating from a dense core (Figure 1D and S2c,d). The needle-like globules (Figures 1D, S1, and S2c,d) and dense granules (Figures S1b and 2a) were found in close proximity to the cells and adjacent to the mature mineral. The dense granules were also dispersed within the mature mineral (Figure S2b), which may represent more mature form of this structure; for this reason, all subsequent nanoanalysis was only performed on the dense clusters adjacent to the mature mineral. The diameter size was significantly different between the dense granules and needle-like globules as shown in Figure S3. Finally, a fourth but less dominant morphology was also observed: calcium phosphate needles emanating from a dense core (Figure 1F). Mineral bodies, termed mineral-bearing globular entities with similar morphologies and size, have previously been identified using cryo-SEM of mineralizing bone in zebrafish fin rays. (2) These bodies have also been shown to be composed of noncrystalline subparticles in a zebra fish model, although direct evidence that these subparticles are amorphous was not provided.(2) Though this needle-like morphology has been observed before,(9) we are not ruling out the possibility that some of the mineral clusters may actually be thin plates rather than "needles". Plates can be difficult to observe in the electron microscope, giving insufficient contrast, unless they are lying edge on to the electron beam. As a result, the terminology of "needle" has been used throughout this report, as that is what is observed experimentally. 
Next, we analyzed the crystallinity of the dense granules and mineral crystals emanating from foci. HRTEM images of the later structures showed regions of crystalline order, approximately a few nanometers in size (Figure S4a,b). The bulk of the dense granules was amorphous and the surface was crystalline (probably an artifact of sample preparation, discussed in SI). In previous work, we also observed mineral crystals emanating from foci. (16) In that case, confined packets of calcium phosphate, obtained from an anhydrous preparation route, were studied by SAED. The SAED showed that the calcium phosphate did not exhibit the expected textured diffraction pattern associated with the mature mineral; regions with crystalline order of the order of a few nanometers were also seen in the material within the vesicles using higher resolution phase contrast imaging.(16) The recent identification of highly disordered $\mathrm{CaP}$ phases, composed of a mixture of highly disordered OCP-like mineral and ACP, as possible transient mineral precursor $(20,21)$ could explain the detection of some order of crystallinity within the dense granules. Poorly crystalline mature aligned mineral associated with the collagenous ECM was also found (Figure 1E). Taken together, our findings support the notion that there is a progression of maturation of the mineral: in the ECM, a highly disordered $\mathrm{CaP}$ transient precursor could be converted to crystalline apatite via an intermediate phase composed of globules of $\mathrm{CaP}$ comprised of crystalline needles, where a dense core acts as a seed for $\mathrm{CaP}$ growth. To confirm this hypothesis, we next examined the chemical composition of each of the mineral morphologies in the ECM.

\section{Chemical composition analyses demonstrate variations in $\mathrm{Ca} / \mathrm{P}$ of mineral morphologies indicating different stages of maturity}

To further elucidate whether the chemistry differs between each of the mineral morphologies and to provide further insight about how the mineral nucleates and grows as it matures, we performed EEL nanospectroscopy to map the chemical composition, bonding, and coordination environment of atoms in the three different mineral morphologies. The electron probe is moved serially across the sample and an EEL spectrum is recorded at each probe position, thus forming a "spectrum image" from which chemical information can be extracted. In all morphologies, we confirmed the presence, in varying amounts, of phosphorus (through its L2,3 ionization edge), calcium (L2,3 edge), nitrogen (K edge), and oxygen (K edge). Typical EEL spectra can be seen in Figure 2A-D, and maps showing the localization of the Ca signal are shown in Figures 3A-C. We have included the bond assignments for each of these edges in Table $\mathrm{S} 1$ based on known energy losses for these bonds. We found no changes in fine structure of the calcium (L2,3), nitrogen (K), and oxygen $(\mathrm{K})$ edges between each dominant $\mathrm{CaP}$ structure (discussed in $\mathrm{SI}$ ).

To test the hypothesis that the different mineral morphologies represent mineral at different stages of maturation, we characterized the $\mathrm{Ca} / \mathrm{P}$ ratios from energy dispersive $\mathrm{X}$-ray (EDX) spectra. The different mineral morphologies had a $\mathrm{Ca} / \mathrm{P}$ with a range of $1.2 \pm 0.3$ to $1.6 \pm 0.2$ (Figure 2E). The dense granules had a $\mathrm{Ca} / \mathrm{P}$ of 1.2 (standard deviation of 0.3 ) that was statistically lower than the needle-like globules (ratio of 1.6; standard deviation of 0.2 ) and mature crystallites (ratio of 1.5; standard deviation of 0.2 ). The $\mathrm{Ca} / \mathrm{P}$ measured for the dense granules is within the range (1.2-2.2) measured for ACP and OCP ( 1.3) and the other two morphologies have a $\mathrm{Ca} / \mathrm{P}$ consistent with calcium deficient hydroxyapatite mineral.(26, 27) 
Using an ex vivo mouse model, Mahamid et al. found values of $\mathrm{Ca} / \mathrm{P}$ of $\sim 1.58$ for mature ECM and $\sim 0.75$ for the intracellular mineral-containing vesicles that were hypothesized to be the precursor of the mature material.(4) The same group has further identified, using EDX of extracted mineral, globular entities within the extracellular matrix with $\mathrm{Ca} / \mathrm{P}$ equal to, or larger than, 1.3,(3) which corroborates our findings and suggests that the apatitic mineral precursor phases may evolve depending on their location (intra- vs extracellular), as previously proposed. $(18,19)$ Although it is not excluded that the dense granule $\mathrm{Ca} / \mathrm{P}$ may be affected by sample preparation, the trend of increasing $\mathrm{Ca} / \mathrm{P}$ as bone mineral matures was observed in both Mahamid's studies $(3,4)$ and in the current work. Together, these data indicate that the $\mathrm{Ca} / \mathrm{P}$ increases successively between the dense granules, the needle-like globules, and the mature crystallites formats, which further supports the hypothesis that the former are a transient precursor phase in mineralizing bone.

\section{EEL spectrum imaging demonstrates a dense cluster of intense carbonate signal in the core of the granules which becomes less intense and less localized as the mineral matures}

EELS was used to analyze the local atomic and chemical environment of the different mineral structures. The most striking variations between the different mineral morphologies were observed in the fine structure of the carbon $\mathrm{K}$ edge, which allowed us to study differences in the level of carbonate. It is well known that in vivo bone carbonate content increases as bone matures,(28-30) and we considered that it might be the case that we could track carbonate substitution in the maturation of mineral.

The carbon K edge signal taken from all mineral morphologies comprises a variety of peaks, with two main features consistently found in all spectra: an initial peak at energies ranging between 284.5 and $\sim 288 \mathrm{eV}$, which—depending on the exact functional chemistrysubdivides into separate features, as explained in detail below, and corresponds to $1 \mathrm{~s} \rightarrow \pi^{*}$ electronic transitions; and a broad shoulder at $297.5 \mathrm{eV}$ (labeled peak D in Figure 3D-F) characteristic of $1 \mathrm{~s} \rightarrow \sigma^{*}$ transitions in the carbon-carbon bonding environment.(31) Bond assignments for the carbon $\mathrm{K}$ edge are listed in Table S2. Amorphous carbon spectra characteristically consist of broad $\pi^{*}$ and $\sigma^{*}$ peaks, with an overall edge onset at $284.5 \mathrm{eV}$ (peak A in Figure 3D-F).(32) Further processing of the carbon K edge was performed using multilinear least-squares (MLLS) to facilitate the identification of the smaller carbonate and carbonyl peaks (described in SI). This approach allows us to compare, in detail, the carbon bonding environment in each of the mineral morphologies. Individual EEL spectra taken from the dense granules (Figure 3A,D) and the needle-like globules (Figure 3B,E) showed a shoulder peak at $287.5 \mathrm{eV}$ (peak B) characteristic of the $1 \mathrm{~s} \rightarrow \pi^{*}$ transition of the carbonyl $(\mathrm{R}-\mathrm{C}=\mathrm{O})$ group.(33) In contrast, this specific subfeature of the $\pi^{*}$ peak was not detected in the mature mineral (Figure 3C,F). Another peak detected at $290.2 \mathrm{eV}$ (peak C in Figure 3D-F) is attributed to the $1 \mathrm{~s} \rightarrow \pi^{*}$ transition in the carbonate group $(31,34,35)$ and was detected from all mineral morphologies. The detection of the carbonate group from the mineralized ECM is consistent with our previous study using Raman spectroscopy showing CO32- $\nu 1$ in-plane vibrations in the mineralized ECM of bone nodules formed from mouse osteoblast cultures.(24) 
The carbonate distribution is most conveniently visualized by mapping the relative intensity of peak $\mathrm{C}$ across the data sets through MLLS (Figure 3D-F). These maps reveal a very strong contribution to the overall carbon $\mathrm{K}$ edge signal arising from the carbonate peak in the case of the mineral granule, with a very dense cluster of intense carbonate signal in the core of the granule (Figure 3G). As the mineral matures, the carbonate signal becomes less localized and less intense as the density of the mineral decreases (Figure $3 \mathrm{H}$ and the less dense material in Figure 3C,I; note the difference with the dense granule at the top of Figure 3C). Similar analysis was also carried out for the carbonyl functional group (Figures 3G-I), revealing similar trends and confirming the initial observation that no carbonyl signature is found in the mature mineral.

Higher presence of carbonate in the young in vitro mineral has been seen in previous in vitro studies performed by Rey et al. using bulk techniques (X-ray diffraction and FTIR). $(28,36)$ Our measurements support this data and show that the coordination environment and chemistry differs between granules, the needle-like globules and the mature mineral. These results also support the findings of a recent study that hypothesized that carbonate-rich mineral deposits are the bioseed of the calcium phosphate deposition process.(38)

\section{Each mineral structure co-localized with nitrogen, which is likely the ECM organic constituent}

In addition to mapping specific chemical groups from the carbon $\mathrm{K}$ edge, it is possible to integrate the spectral intensity corresponding to the various chemical elements and similarly display this as a function of probe position. This has allowed us to generate a map of the distribution of calcium and nitrogen within each mineral structure (Figure 4). The nitrogen signal was found to be co-localized to the mineral or was measured from diffuse "cloudy" structures around the mineral, which are likely the ECM organic constituent (Figure 4 G-I). The presence of both the nitrogen and the carbonyl peaks within the granules (Figure 3D, $3 \mathrm{G}, 4 \mathrm{G}$ ) may be the result of proteins present in the early mineral that play a role in mineralization. There are many known proteins that might be present, including those that have been shown to nucleate and stabilize calcium phosphate and hydroxyapatite during crystallization, including (but not limited to) fetuin, osteopontin and osteocalcin.38-40 Proteomic analysis of MVs have revealed many more, 41 all of which may contribute to the carbonyl peak detected.

In the present study, the application of spatially resolved nanoanalytical electron microscopy has distinguished three dominant $\mathrm{CaP}$ structures in mineralizing osteoblast cultures from a mouse model: $<80 \mathrm{~nm}$ dense granules with a low $\mathrm{Ca} / \mathrm{P}$ and crystalline domains; mineral emanating around a focus of calcium phosphate with diameter $>100 \mathrm{~nm}$; and mature mineral, both with statistically higher $\mathrm{Ca} / \mathrm{P}$ compared to that of the dense granules. This observed link between mineral morphology and $\mathrm{Ca} / \mathrm{P}$ supports other studies in the literature. (4) The identification of a set of dominant structures with specific $\mathrm{Ca} / \mathrm{P}$, in our study, can possibly be explained using the in vitro findings from Habraken et al., who have demonstrated, by direct addition of phosphate ions into a calcium-containing solution in the absence of proteins or ECM, that the growth of calcium phosphate proceeded via multiple distinct morphologies, each representing a separate phase.(9) The relatively short length of 
time required for each phase transition ( 1-2 min) when compared to the length of time the structures remained in a specific morphology ( 20-60 min).(9) Many of the submicron granules and globules were interspersed around fibrillar structures containing nitrogen, which are most likely the organic constituents, for example, collagen or noncollagenous proteins. Spatially resolved maps were acquired using EELS showing the distribution of carbonate within each mineral structure. We found that carbonate moieties were located in the middle of the dense granules, which have been suggested as a precursor $\mathrm{CaP}$ phase. This observation suggested that the formation of carbonate may occur early in the $\mathrm{CaP}$ deposition (possibly in matrix vesicles) and supports recent studies providing evidence that calcium carbonate deposits are the bioseed for calcium phosphate deposition processes in bone, a process driven by increased carbonic anhydrase activity.(38) Notably, the enzyme carbonic anhydrase is known to be present in mammals as a component of matrix vesicles in mineralizing bone and, when inhibited, mineralization of bone apatite (predominantly $\mathrm{Ca} 5(\mathrm{PO} 4) 3 \mathrm{OH})$ is reduced. $(37,42,43)$ In contrast, when bone forming cells are exposed to the carbonic anhydrase activators in vitro, the mineralization process onto the osteoblast-like cells is increased.(44-46) These findings have been hypothesized to be due to a role for the enzyme in preventing the acidification of matrix vesicles, which inhibits calcium phosphate nucleation and crystal growth. $(47,48)$ The evidence presented in the current study suggests an additional (but not exclusive) explanation for the presence of carbonic anhydrase in mammalian matrix vesicles postulated by Müller et al.: to provide a source of bicarbonate for the initial deposition of a calcium carbonate bioseed.(37) Supporting this, other studies have shown that stable ACP was produced in the presence of carbonate ions from supersaturated aqueous solutions containing calcium and phosphate.(11, 12) Carbonate has also been shown to be present in matrix vesicles, which are thought to be the initiators of mineralization and which have previously been shown to contain young, amorphous mineral. $(2,49,50)$ Taking the findings of these previous studies together with our observations in the present study, it is possible that the carbonate detected in the middle of our youngest mineral may be a residue of the initial stabilization events during which rapid precipitation of calcium phosphate occurred from supersaturated aqueous solutions. Müller et al. proposed a mineralization mechanism involving calcium carbonate as follows: First, calcium carbonate is deposited onto cell membranes and constitutes a bioseed for mineralization. This process is enzymatically controlled by carbonic anhydrases. Then, inorganic phosphates are released from adjacent cells through the hydrolysis of calcium-polyphosphate polymers by ALP, which inhibit carbonate anhydrase activity and, together with calcium ions, act as substrates for carbonated amorphous calcium phosphate formation. Carbonate ions are proposed to be nonenzymatically exchanged with phosphate during $\mathrm{CaP}$ formation at neutral $\mathrm{pH} .(18,44-$ 46) Interestingly, these authors have also shown that polyphosphates not only are precursors for inorganic phosphate but also contribute to the supply of metabolic energy.(47) Alternatively, this process may occur intracellularly, supporting our previous work(16) and the work of Mahamid et al. showing that the CaP mineral is formed inside the cells; (3) this provides the intriguing hypothesis that mammals may have retained some components of the mineralizing mechanism present in invertebrates, where calcium carbonate is the primary mineral used for protection and support. Further studies investigating temporally mineral compositions of intracellular and exocytosed matrix vesicles may help provide an answer to this question. 
EEL spectra taken from the dense granules adjacent to the mature mineral and the needlelike globules also showed a carbonyl peak and nitrogen peak which may be the result of proteins present in the mineral; the nitrogen and carbonyl signals were colocalized to the mineral or were measured from diffuse "cloudy" structures around the mineral, possibly the organic material. This finding most likely indicates the presence of proteins, which are known to be fundamental players in the formation of mineral in cellular in vitro and in vivo systems. $(38-40,42,43)$

\section{Conclusions}

In conclusion, this work illustrates that carbonate-rich calcium phosphate dense granules are found at the collagen matrix and may act as seed for growth into larger, submicron-sized, globular aggregates of apatite mineral with a different stoichiometry. These globules appear to mineralize the collagen fibrils forming crystalline textured crystals with a higher $\mathrm{Ca} / \mathrm{P}$ and with less intense carbonate component as the mineral phase of bone. We provide evidence that the use of a carbonate rich, $\mathrm{CaP}$ spherical bioseed could be a process by which a soluble $\mathrm{CaP}$ phase is stabilized and delivered to the collagen for subsequent maturation and collagen mineralization. There is growing evidence that mineral-stabilizing proteins may be involved in mineral maturation.(51) Future work using membrane sensitive stains is needed to assess whether the calcium phosphate material is localized within matrix vesicles. Also, such work could be extended to the analysis of in vivo systems. Although in vitro models (like the calvarial osteoblast model utilized in the current study) are excellent controlled systems for studying cellular processes, they do not fully replicate the complexity of the in vivo situation, such as interactions between different cell types and organ systems (e.g., the blood vessel system). Future work may address this by employing similar techniques during bone development or repair. More generally, we suggest that the dramatically improved energy resolution $(<0.015 \mathrm{eV})$ of a new generation of electron microscopes promises to enable a full characterization of the functional chemistry in these complex minerals,(52) both through enhanced resolution at the core edges and through vibrational spectroscopy techniques at spatial resolutions unattainable with other related techniques, such as Raman spectroscopy. Furthermore, these experiments can be efficiently carried out in so-called "aloof" mode, whereby the electron beam never traverses the sample but is sufficiently close to excite vibrational excitations, thus retaining a high level of spatial localization (nanometer-scale) while providing a very efficient means to limit beam damage effects.(53) The prospects of combining very high energy and spatial resolution electron microscopy, or even vibrational spectroscopy at subnm scale, should soon make the direct detection of these proteins eminently feasible.

\section{Experimental Section}

\section{In vitro mineralization}

Following isolation and expansion, neonatal mouse calvarial osteoblasts were seeded on glass coverslips (30 $\left.000 \mathrm{cells} / \mathrm{cm}^{2}\right)$ and mineralization was induced following a wellestablished mineralization protocol.(16, 24, 54) At day 28 of culture, cells were harvested and were prepared following an anhydrous fixation and resin embedding route previously 
reported(16) that allows to preserve the mineral morphology, crystallinity, and chemistry of the cells. Details of the experimental procedure are provided in the SI section.

\section{Electron Microscopy Imaging and Analysis}

Preliminary bright-field TEM imaging of the extracellular mineral deposits was performed on a JEOL 2000FX. Energy dispersive X-ray spectroscopy (EDX) analysis was carried out on a JEOL 2100F. BF-STEM, HAADF-STEM, and STEM-EELS were performed on a Nion UltraSTEM (SuperSTEM, Daresbury, U.K.) operated at $100 \mathrm{kV}$. HRTEM experiments were conducted using an FEI TITAN 80/300 operated at $80 \mathrm{kV}$ (Figure S4). Dose-induced damage experiments on sacrificial regions of the samples, using a distinct spectral feature at $530 \mathrm{eV}$ in the oxygen K EELS fine structure as a damage marker were performed to identify regimes adapted for reliable interpretation of the EEL spectra (Figure S6). Details of the experiments are available in Supporting Information and Methods.

\section{EDX and EELS analyses}

To calculate the $\mathrm{Ca} / \mathrm{P}$, the maximum of the EDX peaks of $\mathrm{Ca}(\mathrm{Ka})$ and $\mathrm{P}(\mathrm{Ka})$ of the sample were processed using the Cliff-Lorimer equation. $(16,32)$ The $\mathrm{K}$ factor was calculated using a carbonated hydroxyapatite (CHA standard). EELS spectrum images were collected from regions of interest with an energy dispersion of $0.5 \mathrm{eV} /$ channel ensuring that edges from all elements of interest were collected in each spectrum and Gatan DigitalMicrograph software was used for STEM image and EELS analysis. Spectra were calibrated, normalized, and background subtracted. CHA was used as reference spectra to identify the $\mathrm{P}, \mathrm{C}, \mathrm{Ca}, \mathrm{N}$, and $\mathrm{O}$ peaks in the acquired data from the sample. $(32,55)$ Carbonate moieties were identified using multi-Gaussian curve fitting using the carbon K edge from the CHA standard spectra as the reference (Figure S5). Maps of the relative strength of the functional groups of interest (carbonyl and carbonate) were generated for each area of interest. Details of the experiments are provided in Supporting Information.

\section{Statistical Analysis}

Statistical differences between data sets were calculated using one-way ANOVA with Bonferroni post hoc test, with a p value of less than 0.05 as statistically significant $(n>3)$. For sampling of morphological features in the ECM, for bright field and STEM imaging, 100 cells were surveyed from three different cultures. For EDX analysis, 8-16 spectra were acquired from each structure.

\section{Supporting Information}

Refer to Web version on PubMed Central for supplementary material.

\section{Acknowledgment}

A.E.P. gratefully acknowledges funding from the European Research Council, project number 257182 (CNTBBB). M.M.S. acknowledges the ERC Seventh Framework Programme Consolidator grant "Naturale CG" under grant agreement no. 616417. H.A. was supported by the Medical Engineering Solutions in Osteoarthritis Centre of Excellence funded by the Wellcome Trust and the Engineering and Physical Sciences Research Council. All authors would like to acknowledge the work of SuperSTEM, the U.K. National Facility for Aberration-Corrected STEM, supported by the Engineering and Physical Sciences Research Council (EPSRC). We would also like to thank Prof. 
Linn Hobbs (O.B.E.), Prof. Serena Best, Dr. Jeremy Skepper, Dr. Natalie Reznikov, Dr. Sergio Bertazzo, Dr. Angela Goode, and Dr. Michal Klosowski for their helpful discussions and contributions. Funding was provided by European Research Council, project number 257182 (CNTBBB); ERC Seventh Framework Programme Consolidator grant "Naturale CG" under grant agreement no. 616417; Medical Engineering Solutions in Osteoarthritis Centre of Excellence funded by the Wellcome Trust and the Engineering and Physical Sciences Research Council; Engineering and Physical Sciences Research Council (EPSRC).

Funding Sources

European Research Council, project number 257182 (CNTBBB); ERC Seventh Framework Programme Consolidator grant "Naturale CG" under grant agreement no. 616417; Medical Engineering Solutions in Osteoarthritis Centre of Excellence funded by the Wellcome Trust and the Engineering and Physical Sciences Research Council; Engineering and Physical Sciences Research Council (EPSRC).

\author{
Abbreviations \\ $\mathbf{C a} / \mathbf{P}$ \\ EELS \\ ACP \\ CaP \\ ECM \\ OCP \\ cryo-SEM \\ HPF \\ FS \\ STEM \\ EELS \\ HRTEM \\ SAED \\ HAADF-STEM \\ MLLS \\ EDX \\ CHA \\ HA \\ calcium to phosphate ratio \\ electron energy loss spectroscopy \\ amorphous calcium phosphate \\ calcium phosphate \\ extracellular matrix \\ octacalcium phosphate \\ cryo-scanning electron microscopy \\ high pressure freezing \\ freeze substitution \\ scanning transmission electron microscopy \\ electron energy-loss spectroscopy \\ high-resolution transmission electron microscopy \\ selected area electron diffraction \\ high-angle annular dark field scanning transmission \\ electron microscopy \\ multi-linear least squares \\ energy dispersive X-ray \\ carbonated hydroxyapatite \\ hydroxyapatite
}

\title{
References
}

1. Lee DD, Glimcher MJ. 3-Dimentional Spacial Relationship Between the Collagen Fibrils and the Inorganic Calcium-Phosphate Crystals of Pickerel (Americanus-Americanus) and Herring (ClupeaHarengus) Bone. J Mol Biol. 1991; 217:487-501. [PubMed: 1994036] 
2. Mahamid J, Aichmayer B, Shimoni E, Ziblat R, Li C, Siegel S, Paris O, Fratzl P, Weiner S, Addadi L. Mapping Amorphous Calcium Phosphate Transformation into Crystalline Mineral from The Cell to The Bone in Zebrafish Fin Rays. Proc Natl Acad Sci U S A. 2010; 107:6316-21. [PubMed: 20308589]

3. Mahamid J, Sharir A, Addadi L, Weiner S. Amorphous Calcium Phosphate is a Major Component of the Forming Fin Bones of Zebrafish: Indications for an Amorphous Precursor Phase. Proc Natl Acad Sci U S A. 2008; 105:12748-12753. [PubMed: 18753619]

4. Mahamid J, Sharir A, Gur D, Zelzer E, Addadi L, Weiner S. Bone Mineralization Proceeds Through Intracellular Calcium Phosphate Loaded Vesicles: A Cryo-Electron Microscopy Study. J Struct Biol. 2011; 174:527-35. [PubMed: 21440636]

5. Grynpas MD, Omelon S. Transient Precursor Strategy or Very Small Biological Apatite Crystals? Bone. 2007; 41:162-4. [PubMed: 17537689]

6. Beniash E, Metzler RA, Lam RS, Gilbert PU. Transient Amorphous Calcium Phosphate in Forming Enamel. J Struct Biol. 2009; 166:133-43. [PubMed: 19217943]

7. Lowenstam HA, Weiner S. Transformation of Amorphous Calcium-Phosphate to Crystalline Dahllite in The Radular Teeth of Chitons. Science. 1985; 227:51-53. [PubMed: 17810022]

8. Stricker SA, Weiner S. Amorphous Calcium-Phosphate in the Stylets Produced by a Marine Worm (Nemertea). Experientia. 1985; 41:1557-1559.

9. Habraken WJ, Tao J, Brylka LJ, Friedrich H, Bertinetti L, Schenk AS, Verch A, Dmitrovic V, Bomans PH, Frederik PM, Laven J, et al. Ion-Association Complexes Unite Classical and NonClassical Theories for The Biomimetic Nucleation of Calcium Phosphate. Nat Comm. 2013:1507.

10. Wang CG, Liao JW, Gou BD, Huang J, Tang RK, Tao JH, Zhang TL, Wang K. Crystallization at Multiple Sites Inside Particles of Amorphous Calcium Phosphate. Cryst Growth Des. 2009; 9:2620-2626.

11. Bachra BN. Precipitation of Calcium Carbonates and Phosphates from Metastable Solutions. Ann NY Acad Sci. 1963; 109:251-5. [PubMed: 13965921]

12. Bachra BN, Trautz OR, Simon SL. Precipitation of Calcium Carbonates and Phosphates. I. Spontaneous Precipitation of Calcium Carbonates and Phosphates Under Physiological Conditions. Arch Biochem Biophys. 1963; 103:124-38. [PubMed: 14065965]

13. Nudelman F, Pieterse K, George A, Bomans PHH, Friedrich H, Brylka LJ, Hilbers PAJ, de With G, Sommerdijk NAJM. The Role of Collagen in Bone Apatite Formation in The Presence of Hydroxyapatite Nucleation Inhibitors. Nat Mater. 2010; 9:1004-1009. [PubMed: 20972429]

14. Bergen AAB, Plomp AS, Schuurman EJ, Terry S, Breuning M, Dauwerse H, Swart J, Kool M, van Soest S, Baas F, ten Brink JB, et al. Mutations in ABCC6 Cause Pseudoxanthoma Elasticum. Nat Genet. 2000; 25:228-231. [PubMed: 10835643]

15. Murshed M, Harmey D, Millan JL, McKee MD, Karsenty G. Unique Coexpression in Osteoblasts of Broadly Expressed Genes Accounts for The Spatial Restriction of ECM Mineralization to Bone. Gene Dev. 2005; 19:1093-1104. [PubMed: 15833911]

16. Boonrungsiman S, Gentleman E, Carzaniga R, Evans ND, McComb DW, Porter AE, Stevens MM. The Role of Intracellular Calcium Phosphate in Osteoblast-Mediated Bone Apatite Formation. Proc Natl Acad Sci U S A. 2012; 109:4170-5.

17. Xiaohong WE, Schröder HC, Müller W. Polyphosphate as a Metabolic Fuel in Metazoa: A Foundational Breakthrough Invention for Biomedical Applications. Biotechnol J. 2016; 11:11-30. [PubMed: 26356505]

18. Müller W, Tolba E, Heinz C, Schröder HC, Xiaohong WE. Polyphosphate: A Morphogenetically Active Implant Material Serving as Metabolic Fuel for Bone Regeneration Macromolecular Macromol. Biosci. 2015; 15:1182-1197.

19. Omelon S, Georgiou J, Henneman ZJ, Wise LM, Sukhu B, Hunt T, Wynnyckyj C, Holmyard D, Bielecki R, Grynpas MD. Control of Vertebrate Skeletal Mineralization by Polyphosphates. PloS One. 2009; 4:5634.

20. Akiva A, Malkinson G, Masic A, Kerschnitzki M, Bennet M, Fratzl P, Addadi L, Weiner S, Yaniv K. On The Pathway of Mineral Deposition in Larval Zebrafish Caudal Fin Bone. Bone. 2015; 75:192-200. [PubMed: 25725266] 
21. Crane NJ, Popescu V, Morris MD, Steenhuis P, Ignelzi MA Jr. Raman Spectroscopic Evidence for Octacalcium Phosphate and Other Transient Mineral Species Deposited During Intramembranous Mineralization. Bone. 2006; 39:434-42. [PubMed: 16627026]

22. Landis WJ, Glimcher MJ. Electron Diffraction and Electron Probe Microanalysis of The Mineral Phase of Bone Tissue Prepared by Anhydrous Techniques. J of Ultrast Res. 1978; 63:188-223.

23. Landis WJ, Paine MC, Glimcher MJ. Electron Microscopic Observations of Bone Tissue Prepared Anhydrously in Organic Solvents. J Ultrastr Res. 1977; 59:1-30.

24. Gentleman E, Swain RJ, Evans ND, Boonrungsiman S, Jell G, Ball MD, Shean TAV, Oyen ML, Porter A, Stevens MM. Comparative Materials Differences Revealed in Engineered Bone as a Function of Cell-Specific Differentiation. Nat Mater. 2009; 8:763-770. [PubMed: 19633661]

25. Elliott, JC., Dowker, SEP. Apatite Structures. Advances in X-Ray Analysis. Vol. 45. JCPDSInternational Centre for Diffraction Data; 2002.

26. Dorozhkin SV. Calcium Orthophosphates: Occurrence, Properties, Biomineralization, Pathological Calcification and Biomimetic Applications. Biomatter. 2011; 1:121-64. [PubMed: 23507744]

27. Koutsopoulos S. Synthesis and Characterization of Hydroxyapatite Crystals: A Review Study on The Analytical Methods. J Biomed Mater Res. 2002; 62:600-612. [PubMed: 12221709]

28. Rey C, Kim HM, Gerstenfeld L, Glimcher MJ. Structural and Chemical Characteristics and Maturation of the Calcium-Phosphate Crystals Formed During The Calcification of the Organic Matrix Synthesized By Chicken Osteoblasts in Cell Culture. J Bone Miner Res. 1995; 10:1577-88. [PubMed: 8686515]

29. Burnell JM, Teubner EJ, Miller AG. Normal Maturational Changes in Bone Matrix, Mineral, and Crystal Size in the Rat. Calcif Tissue Int. 1980; 31:13-9. [PubMed: 6770970]

30. Legros R, Balmain N, Bonel G. Age-Related Changes in Mineral of Rat and Bovine Cortical Bone. Calcif Tissue Int. 1987; 41:137-44. [PubMed: 3117340]

31. Garvie LAJ, Craven AJ, Brydson R. Use of Electron-Energy-Loss Near-Edge Fine-Structure in The Study of Minerals. Am Mineral. 1994; 79:411-425.

32. Klosowski M, Friederichs RJ, Nichol R, Antoilin N, Carzaniga R, Windl W, Best SM, Shefelbine $\mathrm{S}, \mathrm{McComb}$ D, Porter A. Probing Carbonate in Bone Forming Minerals on The Nanometre Scale. Acta Biomater. 2015; 20:129-39. [PubMed: 25848725]

33. Urquhart SG, Ade H. Trends in The Carbonyl core (C 1s, O 1s) -> pi*c=o Transition in The NearEdge X-ray Absorption Fine Structure Spectra of Organic Molecules. J Phys Chem B. 2002; 106:8531-8538.

34. Brandes JA, Wirick S, Jacobsen C. Carbon K-Edge Spectra of Carbonate Minerals. J Synchrotron Radiat. 2010; 17:676-82. [PubMed: 20724789]

35. Metzler RA, Abrecht M, Olabisi RM, Ariosa D, Johnson CJ, Frazer BH, Coppersmith SN, Gilbert PUPA. Architecture of Columnar Nacre, and Implications for Its Formation Mechanism. Phys Rev Lett. 2007; 98

36. Rey C, Kim HM, Gerstenfeld L, Glimcher MJ. Characterization of The Apatite Crystals of Bone and Their Maturation in Osteoblast Cell Culture: Comparison with Native Bone Crystals. Connect Tissue Res. 1996; 35:343-349. [PubMed: 9084674]

37. Müller WEG, Schroder HC, Schlossmacher U, Grebenjuk VA, Ushijima H, Wang XH. Induction of Carbonic Anhydrase in SaOS-2 Cells, Exposed to Bicarbonate and Consequences for Calcium Phosphate Crystal Formation. Biomaterials. 2013; 34:8671-8680. [PubMed: 23953824]

38. Neuman W, Neuman M, Diamond A, Menanteau J, Gibbons W. Blood:Bone Disequilibrium. VI. Studies of The Solubility Characteristics of Brushite: Apatite Mixtures and Their Stabilization by Noncollagenous Proteins of Bone. Calcif Tissue Int. 1982; 34:149-157. [PubMed: 6805920]

39. Doi Y, Horiguchi T, Kim SH, Moriwaki Y, Wakamatsu N, Adachi M, Ibaraki K, Moriyama K, Sasaki S, Shimokawa H. Effects of Noncollagenous Proteins on The Formation of Apatite in Calcium Beta-Glycerophosphate Solutions. Arch Oral Biol. 1992; 37:15-21. [PubMed: 1596204]

40. Boskey AL, Maresca M, Ullrich W, Doty SB, Butler WT, Prince CW. Osteopontin-Hydroxyapatite Interactions In-Vitro - Inhibition of Hydroxyapatite Formation and Growth in a Gelatin-Gel. Bone Miner. 1993; 22:147-159. [PubMed: 8251766] 
41. Balcerzak M, Malinowska A, Thouverey C, Sekrecka A, Dadlez M, Buchet R, Pikula S. Proteome Analysis of Matrix Vesicles Isolated from Femurs of Chicken Embryo. Proteomics. 2008; 8:192205. [PubMed: 18095356]

42. Stechschulte DJ Jr, Morris DC, Silverton SF, Anderson HC, Vaananen HK. Presence and Specific Concentration of Carbonic Anhydrase II in Matrix Vesicles. Bone Miner. 1992; 17:187-91. [PubMed: 1611308]

43. Anderson HC. Molecular-Biology of Matrix Vesicles. Clin Orthop Relat Res. 1995:266-280.

44. Wang XH, Schröder HC, Schlössmacher U, Neufurth M, Geng Q, Diehl-Seifert B, Müller WEG. Modulation of The Initial Mineralization Process of SaOS-2 Cells by Carbonic Anhydrase Activators and Polyphosphate. Calcif Tissue Int. 2014; 94:495-509. [PubMed: 24374859]

45. Müller W, Schröder H, Wang X. Enzyme-Based Biosilica and Biocalcite: Biomaterials for The Future in Regenerative Medicine. Trends Biotech. 2014; 32:441-147.

46. Müller W, Neufurth M, Huang J, Wang K, Feng Q, Heinz C, Schröder HC, Tolba E, Diehl-Seifert B, Munoz E, Wang X. Nonenzymatic Transformation of Amorphous $\mathrm{CaCO}_{3}$ into Calcium Phosphate Mineral after Exposure to Sodium Phosphate in Vitro: Implications for in Vivo Hydroxyapatite Bone Formation. ChemBioChem. 2015; 16:1323-1332. [PubMed: 25871446]

47. Müller W, Schröder HC, Tolba E, Diehl-Seifert B, Wang X. Mineralization of Bone-Related SaOS-2 Cells under Physiological Hypoxic Conditions. FEBS Journal. 2016; 283:74-87. [PubMed: 26453899]

48. Sauer GR, Genge BR, Wu LN, Donachy JE. A Facilitative Role for Carbonic Anhydrase Activity in Matrix Vesicle Mineralization. Bone Miner. 1994; 26:69-79. [PubMed: 7950506]

49. Chang XT, Zheng YB, Yang QR, Wang L, Pan JH, Xia YF, Yan XF, Han JX. Carbonic Anhydrase I (CA1) is Involved in The Process of Bone Formation and is Susceptible To Ankylosing Spondylitis. Arthritis Res Ther. 2012; 14

50. Wu CY, Martel J, Cheng WY, He CC, Ojcius DM, Young JD. Membrane Vesicles Nucleate Mineral-Organic Nanoparticles and Induce Carbonate Apatite Precipitation in Human Body Fluids. J Biol Chem. 2013; 288:30571-30584. [PubMed: 23990473]

51. George A, Veis A. Phosphorylated Proteins and Control Over Apatite Nucleation, Crystal Growth, and Inhibition. Chem Rev. 2008; 108:4670-93. [PubMed: 18831570]

52. Krivanek OL, Lovejoy TC, Dellby N, Aoki T, Carpenter RW, Rez P, Soignard E, Zhu J, Batson PE, Lagos MJ, Egerton RF, et al. Vibrational Spectroscopy in the Electron Microscope. Nature. 2014; 514:209-12. [PubMed: 25297434]

53. Egerton RF. Vibrational-loss EELS and The Avoidance of Radiation Damage. Ultramicroscopy. 2015; 159:95-100. [PubMed: 26340606]

54. Malaval L, Liu F, Roche P, Aubin JE. Kinetics of Osteoprogenitor Proliferation and Osteoblast Differentiation in Vitro. J Cell Biochem. 1999; 74:616-27. [PubMed: 10440931]

55. Garvie LAJ. Can Electron Energy-Loss Spectroscopy (EELS) be Used To Quantify Hydrogen in Minerals from The O K edge? Am Mineral. 2010; 95:92-97. 


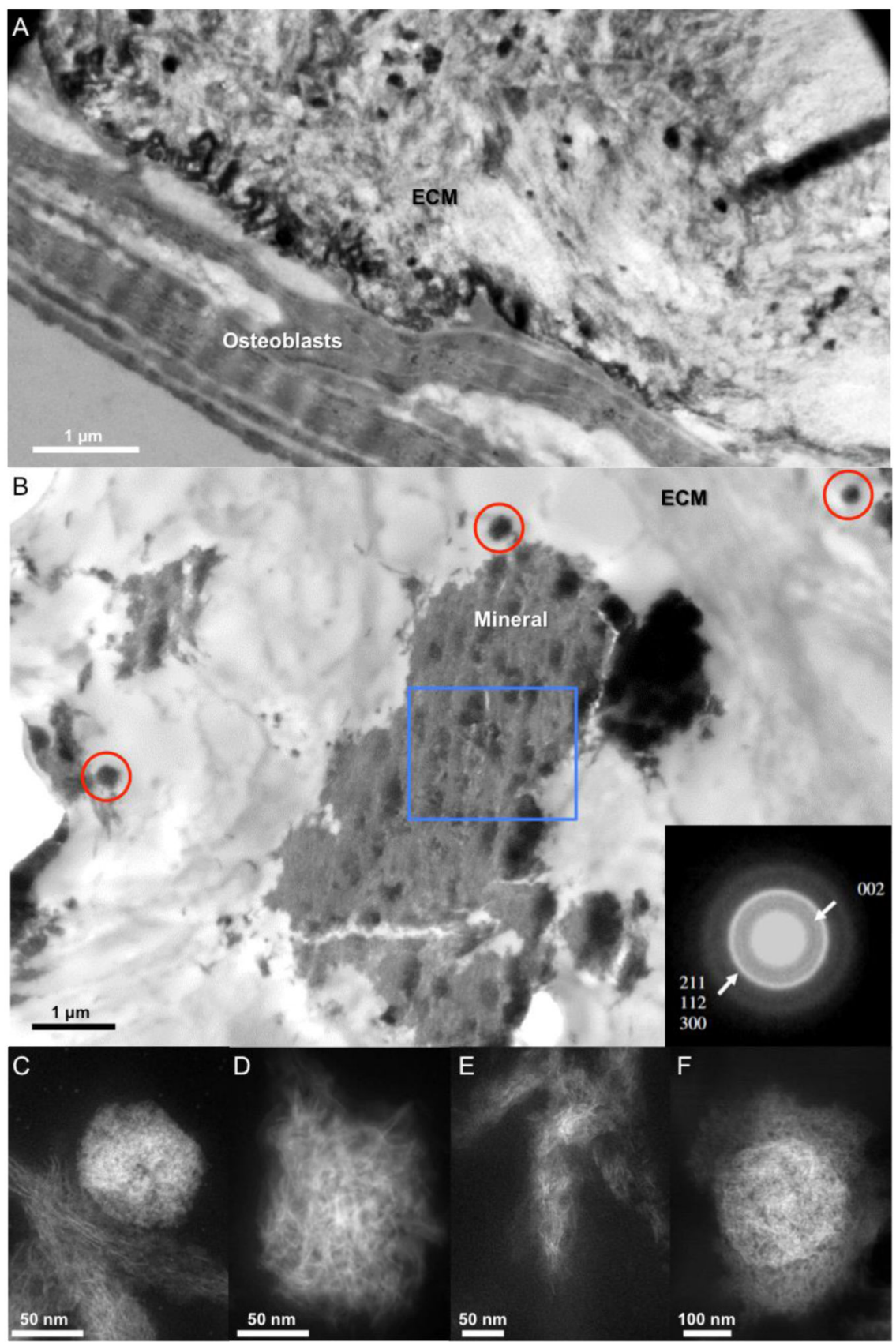

Figure 1. Representative morphologies of the ECM-associated mineral deposits at the nanoscale after 28 days of culture.

(A-B) Low magnification bright field TEM image of mineral deposits formed within the ECM. Samples in (A) were post stained using of uranyl-acetate and lead-citrate to enhance contrast from the cells while no stain was used in (B). SAED pattern (B, inset) of mineralized islets in the ECM (acquired from the large mineral deposit highlighted by the blue box). The red circles in (B) show small mineral clusters. (C-E) High magnification HAADF-STEM images showing the three dominant mineral morphologies found in the ECM. HAADF-STEM image of (C) a dense granule of apatite $<80 \mathrm{~nm}$ diameter (in this case 
co-existing with the mature mineral), (D) a globular calcium phosphate aggregate (>100 nm diameter) comprised of needle-like crystals (with needles of the mineral emanating from a foci), and (E) fibrous mature mineral crystals. (F) Calcium phosphate needles emanating from a dense core. 
A Phosphorous

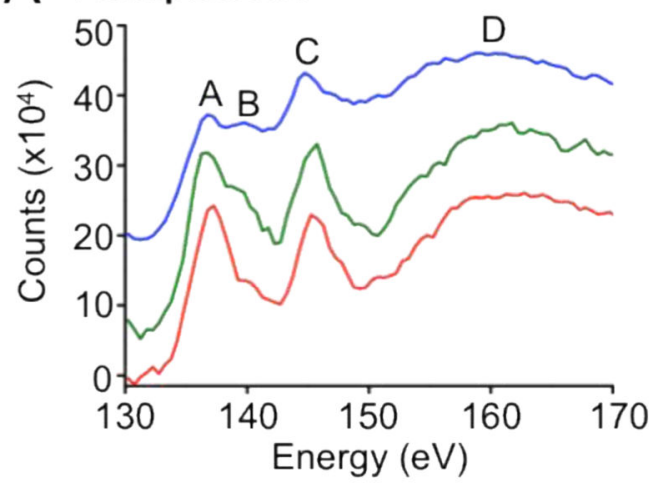

C Nitrogen

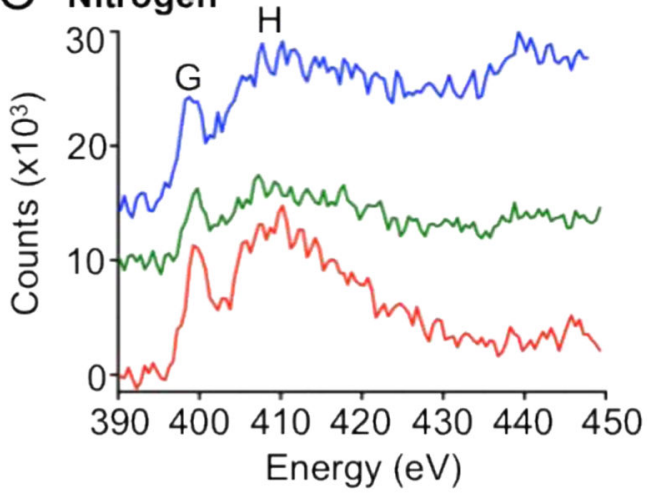

E

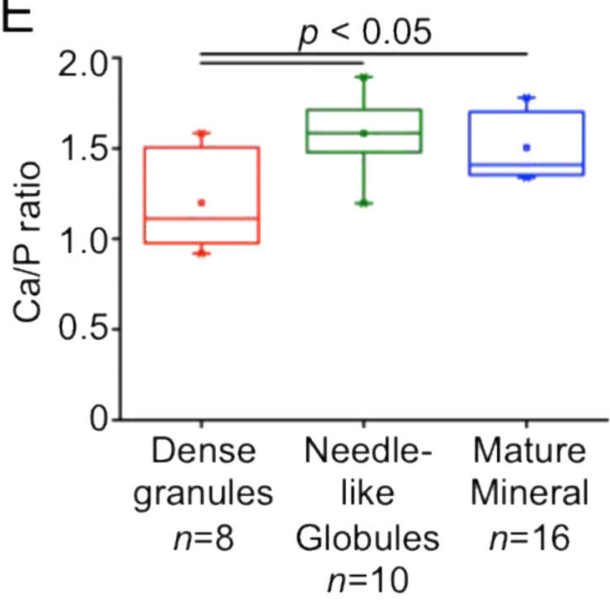

B Calcium

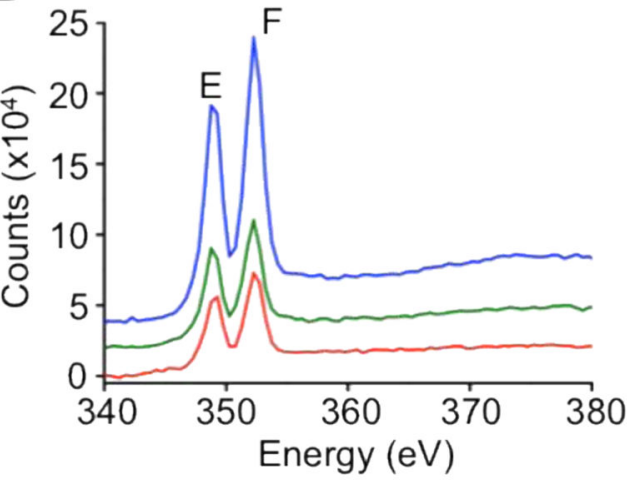

D oxygen

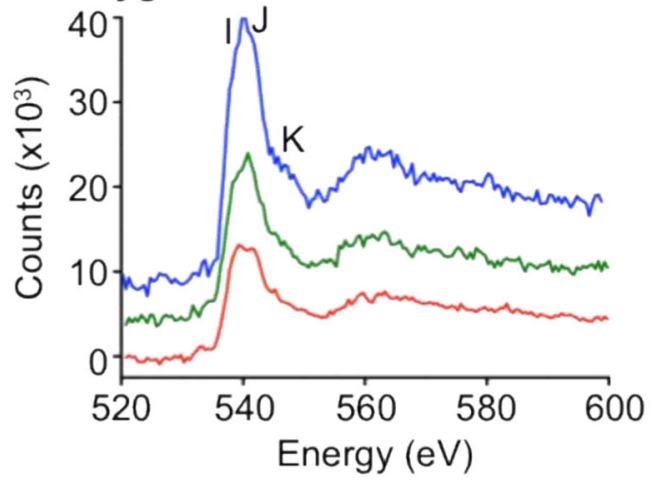

- Dense Granules

— Needle-like Globules

- Mature Mineral

Figure 2. Chemical composition characterization of the three characteristic mineral nanoscale structures.

EEL spectra of the (A) phosphorous $\mathrm{L}_{2,3}$ edge. (B) calcium $\mathrm{L}_{2,3}$ edge, (C) nitrogen $\mathrm{K}$ edge, (D) oxygen $\mathrm{K}$ edge. The peaks (annotated $\mathrm{A}$ to $\mathrm{K}$ within the graphs) are described in Table $\mathrm{S} 1$ and have been assigned according to (33). (E) Variation in $\mathrm{Ca} / \mathrm{P}$ between the distinct mineral deposit morphologies calculated using EDX spectra. The maximum and minimum ranges of the values are denoted by the vertical lines above the box of the box plot. The end whiskers (horizontal line) of the box plot represent the $5^{\text {th }}$ and $95^{\text {th }}$ percentiles, which in this case is also coincides with the $1^{\text {st }}$ and $99^{\text {th }}$ percentiles denoted by $\times$. The box plot itself 
represents the $25^{\text {th }}$ and $75^{\text {th }}$ percentiles with the mean denoted by a small square in the box ( $\square$ ) and the median represented by the horizontal line going through the box. 

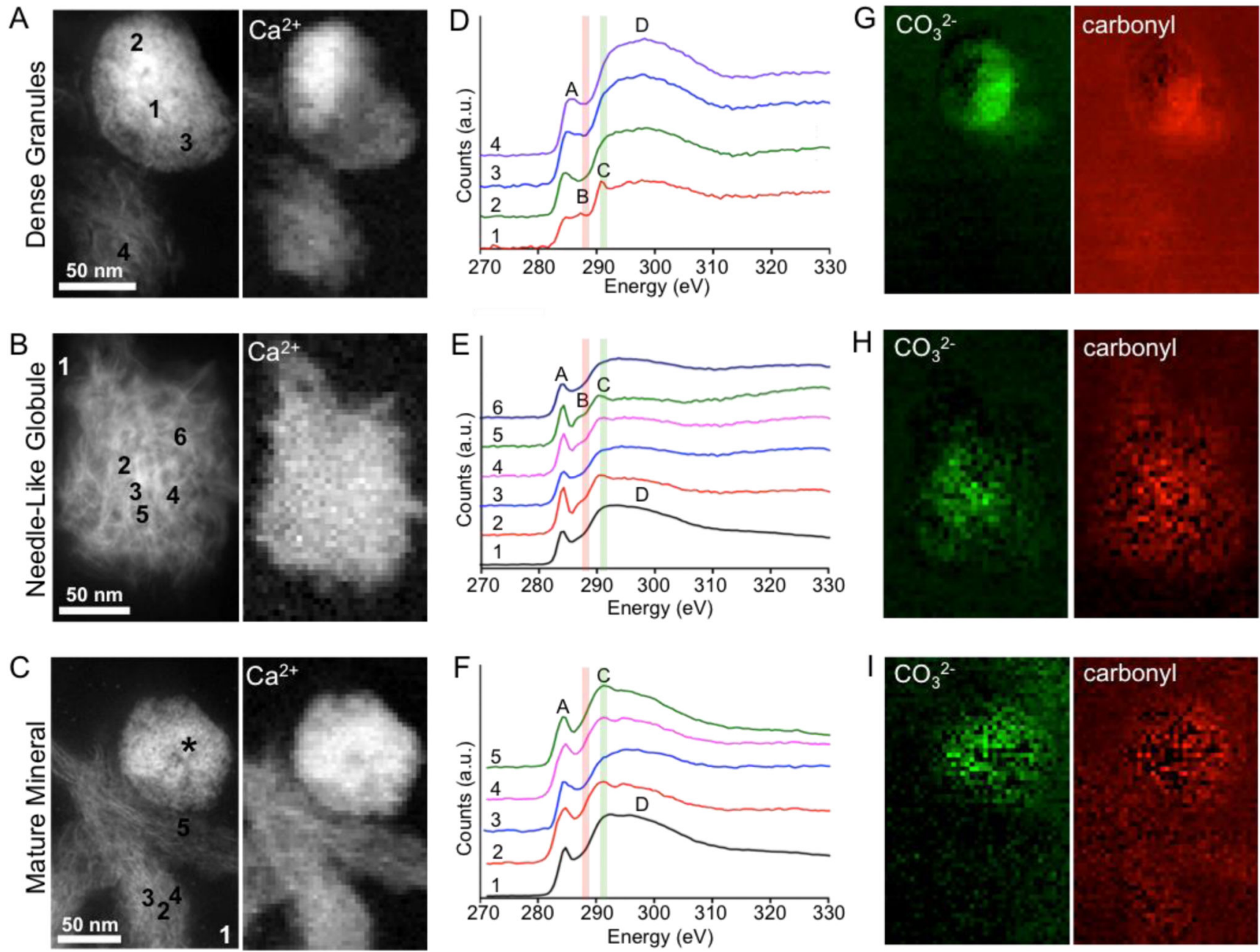

Figure 3. Variation in carbonate content in the different mineral morphologies as demonstrated by HAADF-STEM and EELS.

(A-C) HAADF-STEM images (left) and calcium $\mathrm{L}_{2,3}$ edge intensity heatmaps (right) of (A) a dense granule, (B) a needle-like globule and (C) fibrous mature mineral crystals. The asterisk in (C) indicates a dense granule adjacent to the structure analyzed. (D-F) EEL spectra taken at the carbon K edge from several different pixels within (D) a dense granule, (E) a needle-like globule and (F) fibrous mature mineral crystals. In (D) the spectra were taken from a $5 \times 5$ pixel average to reduce noise. In (E-F) spectra were taken from single pixels and denoised using principal component analysis (PCA, see SI for details). Carbonate and carbonyl groups were characterized by the $290.2 \mathrm{eV}$ peak (peak $\mathrm{C}$, highlighted in green) and the shoulder at $287 \mathrm{eV}$ (peak B and highlighted in red). In (F), the mature needle-like mineral did not contain a carbonyl shoulder in the EEL spectra. Internal EELS references for amorphous carbon were also acquired from the resin-only areas. Assignment of the peaks in the carbon K edge is represented in Table S2. (G-I) Carbonate and carbonyl intensity heatmaps generated through multi-Gaussian fitting for $(\mathrm{G})$ the dense granules, $(\mathrm{H})$ the needle-like globule and (I) the mature mineral. 

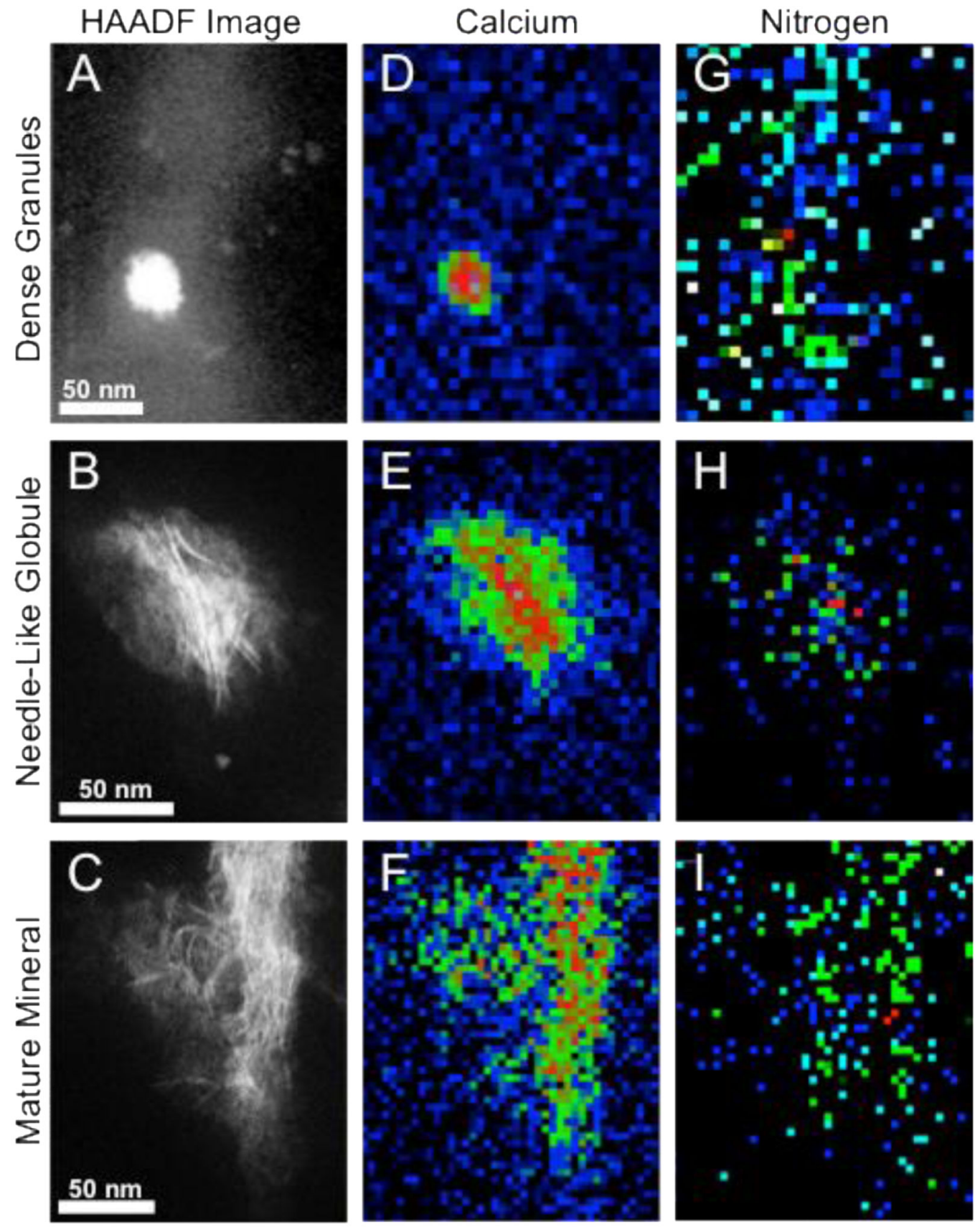

Figure 4. HAADF-STEM and EEL spectrum image suggest the co-localization of nitrogen with the mineral within each morphology.

(A-C) HAADF-STEM images of (A) the dense granules, (B) the needle-like globule and (C) the mature mineral, defining the areas over which the spectrum images were acquired. (D-F) Chemical heatmaps taken at the calcium $\mathrm{L}_{2,3}$ edge of (D) a dense granule, (E) a needle-like globule and (F) fibrous mature mineral crystals. (G-I) Chemical heatmaps taken at the nitrogen $\mathrm{K}$ edge of $(\mathrm{G})$ a dense granule, $(\mathrm{H})$ a needle-like globule and (I) fibrous mature mineral crystals. The calcium $\mathrm{L}_{2,3}$ and nitrogen $\mathrm{K}$ edge intensities were integrated over a 50 $\mathrm{eV}$ window above the edge onset after background subtraction using a power law model. 
The intensity at each pixel is then displayed (after normalization to 1 for convenience) to form a chemical map. 